\title{
ROBUST CONTROL ON HIGH SPEED POSITIONING OF PNEUMATIC SERVO SYSTEM
}

\author{
Hisashi MATSUSHITA and Shujiro DOHTA \\ Department of Mechanical Engineering, Faculty of Engineering \\ Okayama University of Science \\ 1-1, Ridai-cho, Okayama, 700-0005 Japan \\ (E-mail:hisashi@mech.ous.ac.jp)
}

\begin{abstract}
The purpose of this study is to propose a robust control strategy on high-speed positioning of pneumatic servo system, for the changes of system parameters and load mass, and frictional force. Bang-Bang control scheme based on the switching time is one of the most efficient control strategy for high-speed positioning, however this control scheme is influenced by the changes of system parameters and load mass because of open-loop control scheme. In order to overcome these defects, it is introduced a concept of a disturbance observer into Bang-Bang control scheme. That is, the changes of system parameters and load mass can be estimated by a disturbance observer, and the switching time in Bang-Bang control can be modified so as to correspond to the changes of system parameters and load mass, under controlling. It is investigated that the proposed control strategy is effective for the robust high-speed positioning of pneumatic servo system through some experiments.
\end{abstract}

\section{KEY WORDS}

Robust Control, Disturbance Observer, Bang-Bang Control, Pneumatic Servo System, High-Speed Positioning

\section{INTRODUCTION}

In order to improve the performance and function of a pneumatic servo system, some pneumatic servo systems which applied advanced control strategy have been proposed[1,2]. These control strategies are effective when the behavior of controlled object is strictly described with linear mathematical model and the system parameters of that controlled object don't change mainly while the servo system drives. However, in the pneumatic servo system, it is not so easy to identify system parameters and the value of some parameters changes mainly from the identified value. Moreover, it is problem that the control performance using a pneumatic servo system is worse than the control performance using an electric servo system for the delay in response caused by air compressibility and the structure of control valve, Coulomb frictional force in the sliding part of actuator and so on. Therefore, it is strongly desired to establish the control strategy added the robustness for the change of system parameters to the high-speed characteristics of pneumatic servo system.

The purpose of this study is to establish a robust control strategy on high-speed positioning of pneumatic servo system using the pneumatic rodless cylinder, with assuming high-speed conveyance work in the case as the load mass and the frictional force change. As one technique to realize such control strategy, the control strategy that a disturbance observer is added to Bang-Bang control scheme can be proposed. A high-speed positioning is realized by using BangBang control scheme, when paying attention to the point that a pneumatic servo system can be driven 
at high speed in the case of supplying compressed air by on and off drive. Also, a robust positioning is realized by using a disturbance observer for the changes of system parameters and load mass, and for the frictional force and external force[3].

\section{PNEUMATIC SERVO SYSTEM}

\section{Construction}

The configuration of a pneumatic servo system is shown in Figure 1. This system is constructed from a pneumatic rodless cylinder with an effective sectional area of $491 \mathrm{~mm}^{2}$ and full stroke of $1000 \mathrm{~mm}$, two electro-pneumatic proportional pressure control valves, a cart that the mass is $1.7 \mathrm{~kg}$, a rotary type encoder and a personal computer.

The cart position is detected by means of a rotary type encoder connected to the cart through belts and pulleys. This detected signal is fed back to the personal computer through counter. The control input signals obtained in accordance with the control strategy are provided to each of control valves through digital-analog converters and power amplifiers. The compressed air which is proportional to the control input signal is applied to the pneumatic cylinder and the position of the cart is controlled.

In this servo system, the resolution for detecting is $4 \mu \mathrm{m}$ in the cart position, and sampling period for control is $2.5 \mathrm{msec}$.

\section{Modelling}

Generally, dynamic characteristics of a pneumatic servo system shown in Figure 1, i.e. the relation of the cart displacement to the control input voltage to the amplifier which drives control valve is described by the third order transfer function. In this paper, in order to smplify the design of controller, it is approximated as the system which contains dead time element in the control input which is described by

$$
m \ddot{x}(t)+C \dot{x}(t)=R u(t-L)-F_{c} \operatorname{sgn}(\dot{x}(t))
$$

where, $C$ is the viscous friction coefficient of the cart, $F_{c}$ is the Coulomb frictional force, $m$ is the equivalent mass of the cart, $R$ is the gain factor of the servo system, $u(t)$ is the control input voltage to the control valve drive amplifier and $x(t)$ is the cart displacement. $L$ is dead time, and is defined as the time that the cart velocity exceeds $0.1 \mathrm{~m} / \mathrm{s}$ after the stepwise control input impresses. Dead time of this servo system changes into $0.075 \mathrm{sec}$ from $0.040 \mathrm{sec}$ as the load mass increases. Therefore, dead time is identified while controlling.

From equation (1), relation between the input voltage $u$ and steady state velocity $\dot{x}_{s}$ in the case of mov-

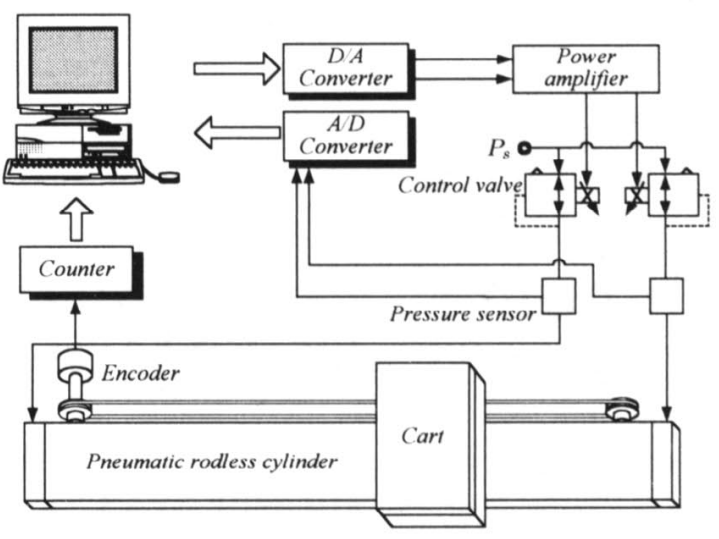

Figure 1 Configuration of pneumatic servo system

ing a cart with uniform motion is written by

$$
\dot{x}_{s}=\frac{R}{C} u-\frac{F_{c}}{C}
$$

From the relation between the input voltage and the cart velocity to some load mass which is obtained through the experiment, the viscous friction coefficient $C$ and Coulomb frictional force $F_{c}$ are identified. As for the gain factor $R, 82 \mathrm{~N} / \mathrm{v}$ is used. Contrary to the expectation, Coulomb frictional force is constant approximately irrespective of the load mass and is identified as $20.4 \mathrm{~N}$. On the other hand, the viscous friction coefficient increases as the load mass increases, the relation between the viscous friction coefficient and reciprocal of load mass is approximated by

$$
C=-\frac{35.7}{m}+82.7 \quad(1.7 \leq m \leq 8.2 \mathrm{~kg})
$$

\section{CONTROL STRATEGY}

\section{Bang-Bang Control}

There are two kinds of techniques to switch control input in Bang-Bang control scheme. One is the switching technique based on the phase plane with displacement and velocity of the controlled object. Another is the technique to switch control input at the time which is calculated based on the mathematical model of the controlled object in advance. In the case that dead time exists in the control input such as this servo system, it is considered that the technique of the latter is more useful, from the viewpoint that the switching of control input is not influenced by the operating delay of the control input in order to switch control input at the time which is decided by the system parameter, than the technique of the 
former. In this study, the switching technique of the latter is used.

When ignoring dead time of the control input $L$ and Coulomb frictional force $F_{c}$ so as to obtain the switching time of the control input in this servo system, equation ( 1 ) is described by

$$
m \ddot{x}(t)+C \dot{x}(t)=R u(t)
$$

From equation (4), the switching times $t_{1}$ and $t_{2}$ are given by

$$
\begin{aligned}
t_{1} & =\frac{m}{C}\{\ln (1+\sqrt{1-\exp \phi})-\phi\} \\
t_{2} & =\frac{m}{C}\{2 \ln (1+\sqrt{1-\exp \phi})-\phi\} \\
\phi & =-\frac{C^{2} x_{0}}{R m u_{m}}
\end{aligned}
$$

where, $u_{m}$ is the control input in Bang-Bang control and $x_{0}$ is the reference position of the cart. As seen from equations (5),(6) and (7), the switching times $t_{1}$ and $t_{2}$ are decided by the system parameters and reference position, and are influenced by the change of system parameters. The technique to cope with this problem is explained at the following paragraphs.

\section{Disturbance Observer}

A disturbance observer can estimate the size of the change from the nominal value of the system parameters of the load mass and the viscous friction coefficient and so on. Because the estimated results are used for the feedback control, the sensitivity to the change of the system parameters can be reduced and the robust controller can be composed for the change of the system parameters.

A block diagram of a proposed robust control system is shown in Figure 2. In this figure, $\hat{d}(s)$ is the estimated disturbance, $P(s)$ is the transfer function of the controlled object, $P_{n}(s)$ is the transfer function of nominal model in the controlled object and $Q(s)$ is the transfer function of filter. In order to compensate for dead time which exists in the control input, a element $e^{-L s}$ is inserted in the feedback loop of the control input. Relation in the broken line shown in Figure 2 is described by

$$
x(s)=\frac{1}{G(s)} u_{1}(s)+\frac{1-e^{-L s} Q(s)}{G(s)} d(s)
$$

where,

$$
G(s)=P^{-1}(s)\left\{1-e^{-L s} Q(s)\right\}+P_{n}^{-1}(s) Q(s)
$$

From the equation of motion (4), $P_{n}(s)$ is given by

$$
P_{n}(s)=\frac{x(s)}{u(s)}=\frac{R}{s\left(m_{n} s+C_{n}\right)}
$$

where, the nominal value of viscous friction coefficient $C_{n}$ and the nominal value of load mass $m_{n}$ are set to $m_{n}=2.2 \mathrm{~kg}$ and $C_{n}=66.5 \mathrm{~kg} / \mathrm{s}$, respectively. From equation (8), the more product $e^{-L s} Q(s)$ between dead time element $e^{-L s}$ and the filter $Q(s)$ approaches 1 , the more control output variable $x(s)$ becomes

$$
x(s) \rightarrow P_{n}(s) e^{-L s} u_{1}(s)
$$

As the switching times $t_{1}$ and $t_{2}$ which are calculated based on $P_{n}(s)$ are a constant value, $x(t)$ is not influenced by the changes of the system parameters and the load mass, and moreover disturbance $d(s)$. The filter $Q(s)$ which has a low pass filtering property is designed by

$$
Q(s)=\frac{1}{\left(1+T_{q} s\right)^{2}}
$$

Also, in order to avoid that the positioning accuracy becomes bad with the influence of the change of the system parameters and Coulomb frictional force, traditional PID controller, too, is added as shown in Figure 2. After passing in dead time $L$ from the switching time $t_{2}$, it is switched to the PID control from Bang-Bang control.

\section{Switching Time Correction}

Because the gain of transfer function $e^{-L s} Q(s)$ can not be made 1 , the change of the system parameters and Coulomb frictional force are not accurately estimated, and the control input voltage which is necessary for the positioning control can not be applied. Therefore, the technique to correct the switching times can be proposed. The state of the control input about each time is shown in Figure 3. The control input which is shown by the solid line with dark color is the ideal control input to estimate the change of the system parameters, Coulomb frictional force and so on. And the control input which is shown by the solid line with light color is actually used control input. The control input which is lacking is equivalent to the area of the part which is surrounded in $u_{1}-\hat{d}$ and $u$ as shown in Figure 3 . Therefore, at every sampling time, the correction time of the switching time is calculated by

$$
\Delta_{1 o r 2}(t)=\frac{1}{u(t)} \int_{0}^{t}\left\{u_{2}(\tau)-\hat{d}(\tau)\right\} d \tau
$$

The robust control is realized by

$$
u(t)= \begin{cases}u_{m}-u_{2}(t) & \left(0 \leq t \leq \tau_{1}\right) \\ -u_{m}-u_{2}(t) & \left(\tau_{1} \leq t \leq \tau_{2}\right) \\ -u_{2}(t) & \left(\tau_{2} \leq t \leq \tau_{3}\right) \\ u_{1}(t)-u_{2}(t) & \left(\tau_{3} \leq t\right)\end{cases}
$$

That is, until time $\tau_{2}=t_{2}+\Delta_{1}+\Delta_{2}$, Bang-Bang control which a disturbance observer is added to is 


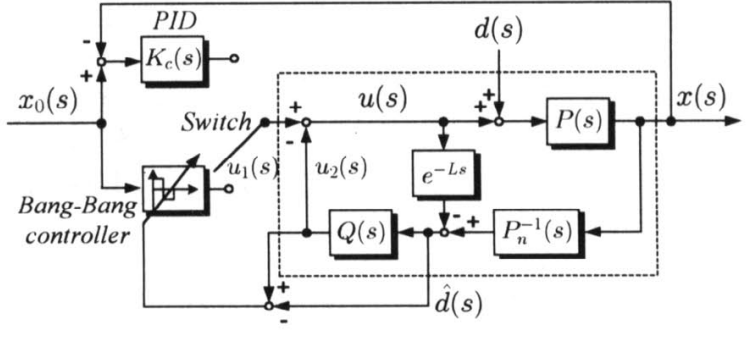

Figure 2 Block diagram of robust control system

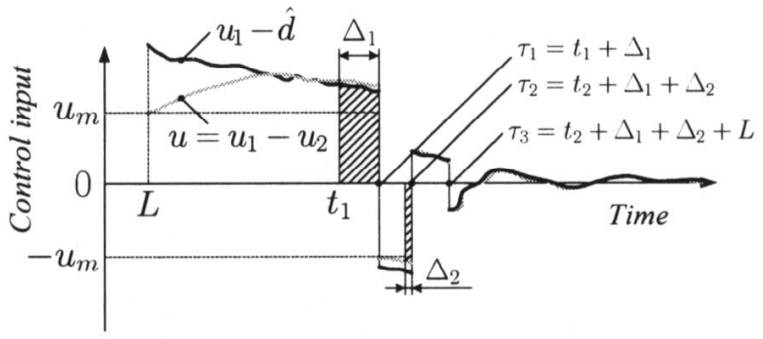

Figure 3 Concept of switching time correction

implemented. And, considering that it is delay time in the control input, it is switched to PID control which a disturbance observer is added to, after dead time $L$ passes.

\section{Controller Design}

At first, the feedback gains of PID controller are designed based on an improved ultimate sensitivity method[4], for the load mass $(7.2 \mathrm{~kg})$ which is the heaviest in the load mass which were used by the experiment, because of the reason for being described below.

Next, the time constant $T_{q}$ of the filter $Q(s)$ which is described by equation (11) is designed based on Nyquist stability criterion. According to the results that investigated the vector loci about open-loop transfer function of the robust control system shown in Figure 2, it is found that the more the load mass increases, the lower the degree of stability in this servo system becomes. Therefore, a time constant $T_{q}$ should be designed based on the case that the load mass is heavy.

In this study, for the phase margin to become equal to or more than 20deg from the viewpoint with degree of stability when the load mass is $7.2 \mathrm{~kg}$, and for $Q$ to approach 1 in the frequency range which is as wide as possible, $T_{q}=0.07 \mathrm{sec}$ is chosen.

\section{RESULTS AND DISCUSSIONS}

The responses of the cart in the case that the reference position of the cart is changed into $0.8 \mathrm{~m}$ in step-

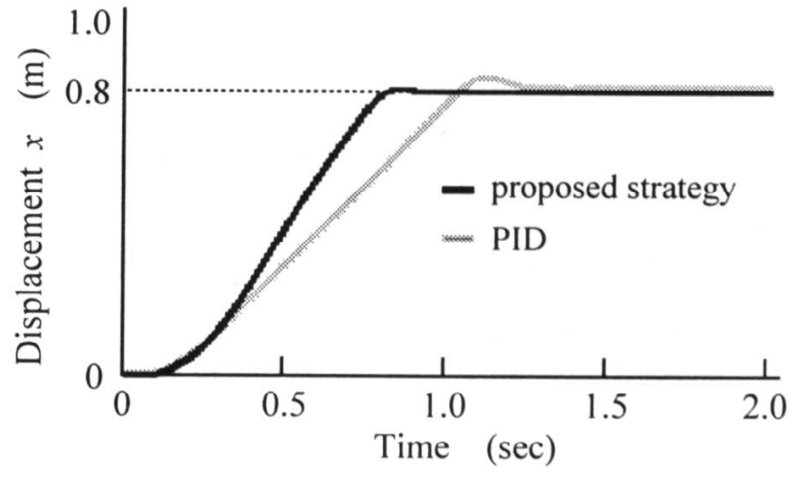

Figure 4 Comparison between proposed robust control and PID control about command response

wise are shown in Figure 4. The load mass is $5.2 \mathrm{~kg}$. The solid line with dark color shows the response in the case of using the proposed robust control strategy. The response in the case of using the traditional PID control method is shown by the solid line with light color, to compare with the proposed robust control strategy. The settling time of the cart is about $0.8 \mathrm{sec}$ in the case of using robust control strategy, and is about 1.3sec in the case of using PID control method. The time when the cart stops within $\pm 5 \%$ of the reference position is defined as settling time. The settling time of the cart with robust control is about $40 \%$ shorter than that of using PID control. Also, in the case of using PID control method, the overshoot is seen. It is clear that the proposed robust control strategy is effective for the high-speed positioning of pneumatic servo system from this experimental result.

Next, the effectiveness of correcting the switching times in Bang-Bang control is investigated. The responses of the cart in the case that the reference position of the cart is changed in stepwise are shown in Figure 5 (a). The reference positions of the cart are $0.2,0.5$ and $0.8 \mathrm{~m}$, respectively. The solid lines with dark color are the responses of the cart with switching time correction, and the solid lines with light color are the responses of the cart without switching time correction. It is found that the cart settles to the reference position without decelerating in front of the reference position to any reference position by using robust control strategy with switching time correction, when comparing with the case of using robust control strategy without switching time correction. Also, as seen from Figure 5 (b) which showed the state of the change of the control input in the case that the reference position is $0.5 \mathrm{~m}$, the switch- 


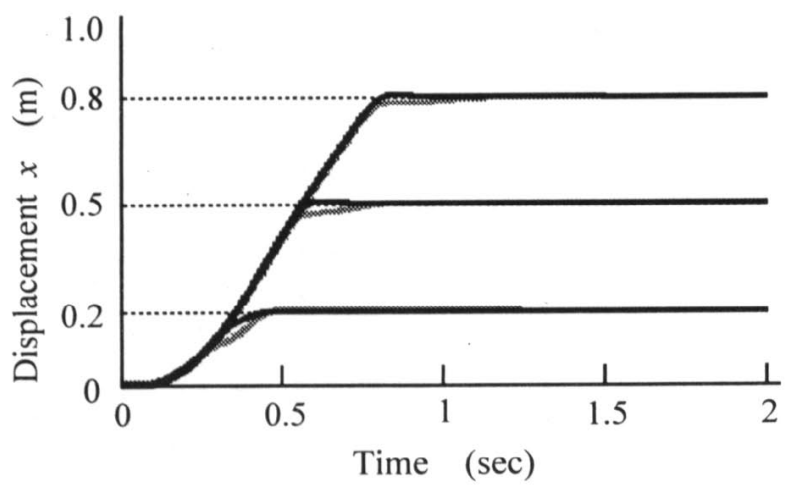

(a) Performance of command responses

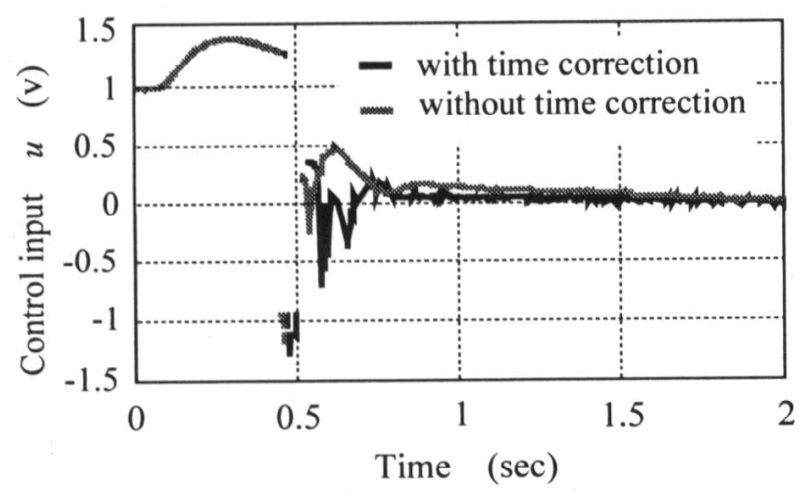

(b) State of control input

Figure 5 Comparison between with switching time correction and without switching time correction

ing times are appropriately corrected in accordance with the technique proposed to correct the switching times. By the reference, in this case, $t_{1}$ is $0.437, t_{2}$ is $0.459, \Delta_{1}$ is 0.0325 and $\Delta_{2}$ is $0.0075 \mathrm{sec}$.

Next, the influence of the change of load mass on the response of the cart is investigated. The responses of the cart in the case that the load mass are 2.2 and $7.2 \mathrm{~kg}$ are shown in Figure 6 (a). The reference positions of the cart are $0.2,0.5$ and $0.8 \mathrm{~m}$, respectively. In spite of changing with 3.3 times of the load mass from its nominal value, the responses of the cart are good to all reference positions. Also, the responses of the cart in the case that the disturbance $d(s)$ is applied into $80 \mathrm{~N}$ in stepwise are shown in Figure 6 (b). The disturbance is applied when the time is $0.5 \mathrm{sec}$ as software disturbance. From this figure, as for the response of the cart about the disturbance, the difference of the response by the change of the load mass is not so seen. And after the distrubance is applied, about $0.6 \mathrm{sec}$ later, the disturbance can be almost suppressed. It is understood that the proposed control strategy has the robustness for the change of

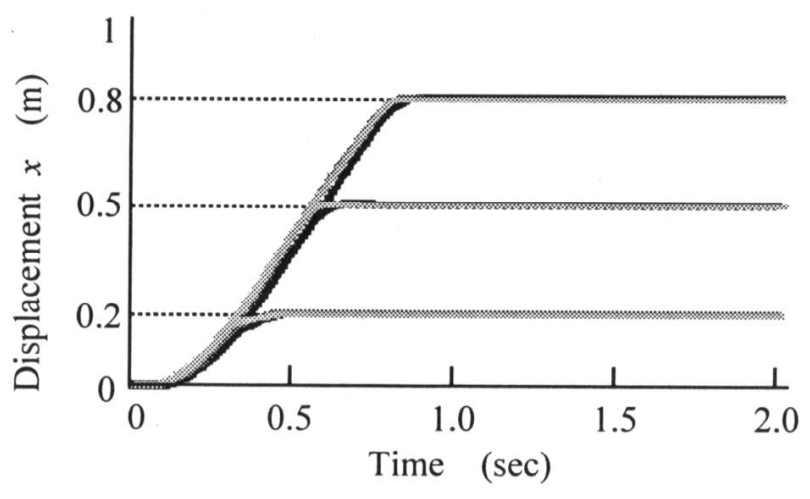

(a) Performance of command responses

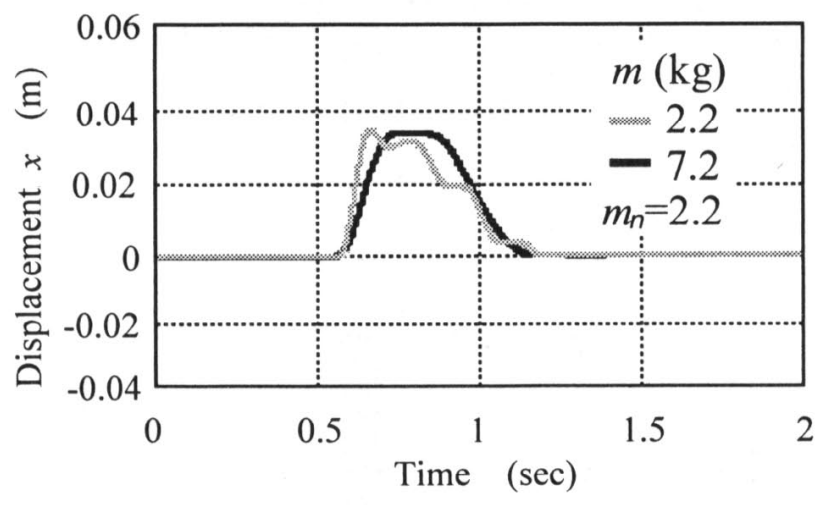

(b) Performance of disturbance suppression

Figure 6 Comparison of responses for load mass

the load mass and disturbance.

The Histogram in the settling time when the positioning control is repeatedly done 30 times is shown in Figure 7. The Histogram when the reference position of the cart is $0.5 \mathrm{~m}$ is shown in (a) of this figure and the histogram when the reference position is $0.8 \mathrm{~m}$ is shown in the same figure (b). The load mass is $5.2 \mathrm{~kg}$. As seen from Figure 7 (a), in the case that the reference position is $0.5 \mathrm{~m}$, the time which is necessary to make a cart settle is among from 0.48 to 0.51 sec. Also, as seen from Figure 7 (b), in the case that the reference position is $0.8 \mathrm{~m}$, the cart is getting to the reference position within approximately $0.8 \mathrm{sec}$, and the average velocity when the cart moves to the reference position is about $1 \mathrm{~m} / \mathrm{sec}$.

Finaly, the positioning accuracy of the cart is examined. The histogram in the steady state error when the positioning control is repeatedly done 30 times is shown in Figure 8. The reference position is $0.5 \mathrm{~m}$, and the load mass is three kinds of $2.2,5.2$ and $7.2 \mathrm{~kg}$. The error between the reference position and the position when passing for $0.4 \mathrm{sec}$ after a cart is settled is defined as steady state error. From this figure, it 


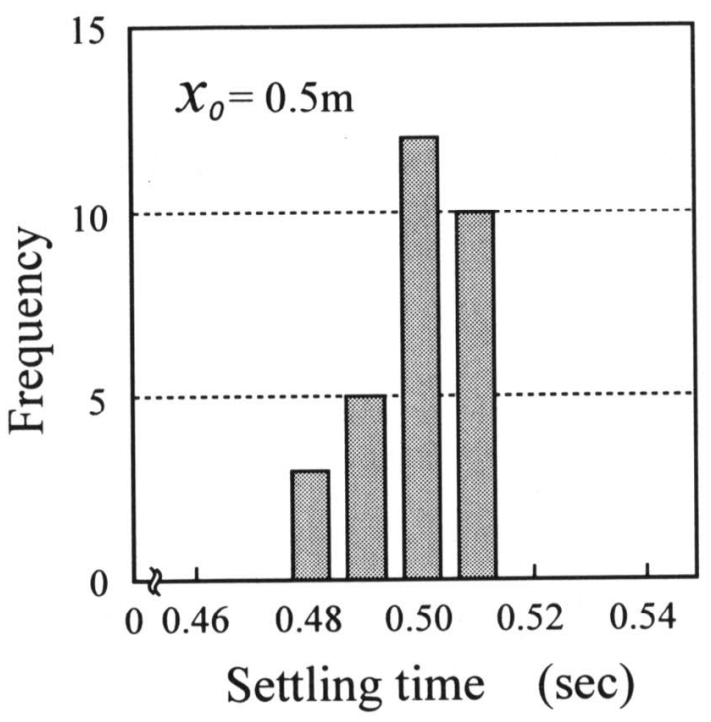

(a) Reference position is $0.5 \mathrm{~m}$

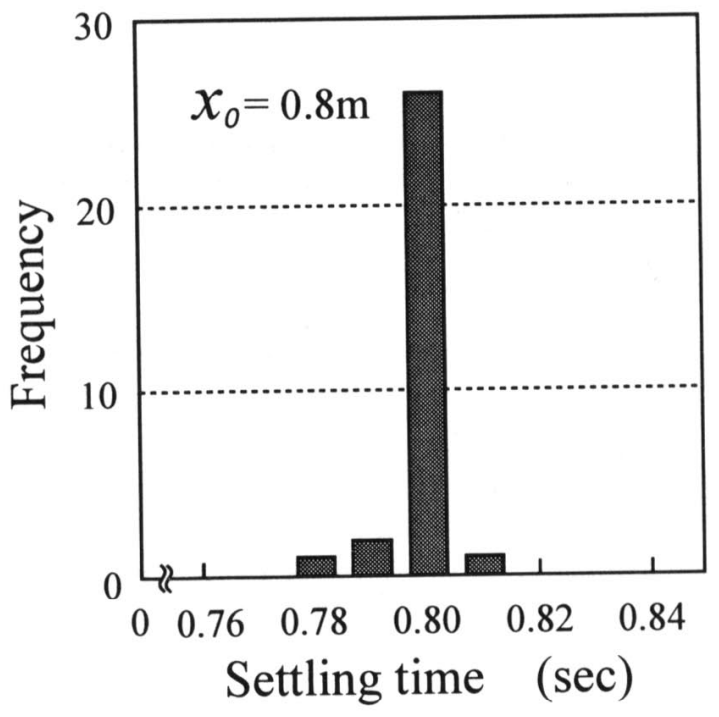

(b) Reference position is $0.8 \mathrm{~m}$

Figure 7 Histogram of settling time

is confirmed that the positioning accuracy is within $\pm 2 \mathrm{~mm}$, irrespective of the weight with the load mass. Therefore, It can be expected that the influences of the change of the load mass on the positioning accurasy in the pneumatic servo system is small with this proposed control strategy.

\section{CONCLUSIONS}

The purpose of this study is to establish the robust control strategy on high-speed positioning of pneumatic servo system for the changes of system param-

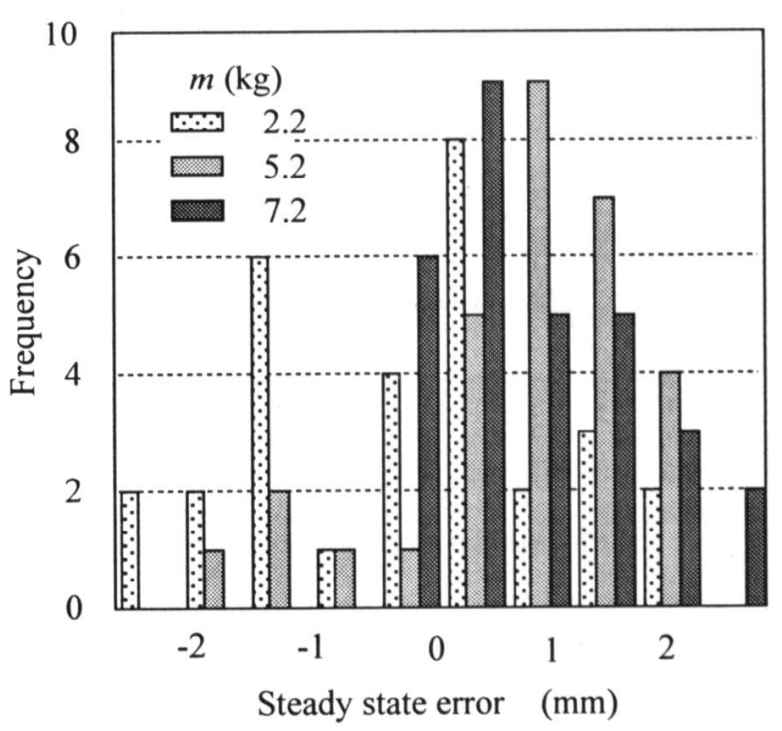

Figure 8 Positioning accuracy for various load mass

eters and load mass, and frictional force and external force, and then, the results of this study is summarized as follows.

- The mathematical model of a pneumatic servo system developed by using a pneumatic rodless cylinder was obtained and the system parameters were identified.

- The robust control system utilized the advantages of a disturbance observer and Bang-Bang control scheme was designed. And a technique to correct the switching times in Bang-Bang control was proposed.

- The effectiveness of the proposed robust control strategy for robust high-speed positioning of pneumatic servo system so that the system parameters and load mass change was confirmed, through some various experiments.

\section{REFERENCES}

1. Oyama, O., "Preview Control of Pneumatic Servo Cylinder", Proc FLUCOME'97, 1997, pp.265-270.

2. Kimura, T., et al., "Sampled-data $\mathrm{H}^{\infty}$ Control for a Pressure Control System Considering Transmission Line Delay", Proc FLUCOME'97, 1997, pp.271-276.

3. Noritsugu, T. and Takaiwa, M., "Robust Positioning Control of Pneumatic Servo System with Pressure Control Loop", Proc IEEE Int Conf on Robotics and Automation, 1995, pp.2613-2618.

4. Kuwata, R., "An Improved Ultimate Sensitivity Method and PID; I-PD Control Characteristics", Trans SICE, 1987, 23-3, pp.232-239. 